\title{
DISTINCTION BETWEEN THE EXTRACELLULAR MATRIX OF THE NUCLEUS PULPOSUS AND HYALINE CARTILAGE: A REQUISITE FOR TISSUE ENGINEERING OF INTERVERTEBRAL DISC
}

\author{
F. Mwale ${ }^{1 *}$, P. Roughley ${ }^{2}$ and J. Antoniou ${ }^{1}$ \\ ${ }^{1}$ Lady Davis Institute for Medical Research and Department of Surgery, SMBD-Jewish General Hospital, Montreal (QC) Canada \\ ${ }^{2}$ Genetics Unit, Shriners Hospitals for Children and Department of Surgery, McGill University, Montreal (QC) Canada
}

\begin{abstract}
Tissue engineering of intervertebral discs (IVD) using mesenchymal stem cells (MSCs) induced to differentiate into a disc-cell phenotype has been considered as an alternative treatment for disc degeneration. However, since there is no unique marker characteristic of discs and since hyaline cartilage and immature nucleus pulposus (NP) possess similar macromolecules in their extracellular matrix, it is currently difficult to recognize MSC conversion to a disc cell. This study was performed to compare the proteoglycan to collagen ratio (measured as GAG to hydroxyproline ratio) in the NP of normal disc to that of the hyaline cartilage of the endplate within the same group of individuals and test the hypothesis that this ratio can be used for in vivo studies to distinguish between a normal NP and hyaline cartilage phenotype. Whole human lumbar spine specimens from fresh cadavers, ranging in age from 12 weeks to 79 years, were used to harvest the IVDs and adjacent endplates. The GAG to hydroxyproline ratio within the NP of young adults is approximately $27: 1$, whereas the ratio within the hyaline cartilage endplate of the same aged individuals is about 2:1. The production of an extracellular matrix with a high proteoglycan to collagen ratio can be used in vivo to distinguish NP cells from chondrocytes, and could help in identifying a NP-like phenotype in vivo as opposed to a chondrocyte when MSCs are induced to differentiate for tissue engineering of a disc.
\end{abstract}

Key Words: Tissue engineering, intervertebral disc, proteoglycan, collagen, glycosaminoglycan, nucleus pulposus, annulus fibrosus, disc degeneration, hyaline cartilage.

\author{
*Address for correspondence: \\ F. Mwale \\ Lady Davis Institute for Medical Research \\ SMBD-Jewish General Hospital, \\ Department of Surgery, \\ McGill University \\ 3755, Chemin de la Cote St. Catherine, LDI 602 \\ Montreal, \\ Quebec, \\ H3T 1E2, Canada \\ Telephone Number: 514-340-8222 ext 2948/5287 \\ FAX Number: 514-340-7502/7573 \\ E-mail: fmwale@ldi.jgh.mcgill.ca
}

\section{Introduction}

Diseases of the spine that afflict the elderly population involve defects in intervertebral disc (IVD) (Seitsalo et al., 1997; Cinotti et al., 2002). Low back pain is a leading cause of morbidity and it is estimated that about $70 \%$ of the population will experience low back pain in their lives (Macfarlane et al., 1999). Although the aetiology of low back pain is often unclear, it is believed that disc degeneration plays a major role (Salminen et al., 1999). While the majority of those affected will not require prolonged medical care or absence from work, about one third will require extensive care involving hospitalization. Present management of disc pathology has been focused on relieving the symptoms caused by degeneration, and much less study has been devoted to disc regeneration.

The surgical treatment of idiopathic low back pain associated with lumbar degenerative disc disease includes disc excision and vertebral fusion (Nakano and Tomita, 1980; Snider et al., 1999). Although surgical procedures produce a good short-term clinical result in relief of pain, they alter the biomechanics of the spine, leading to further degeneration of surrounding tissue and discs at adjacent levels (Lee, 1988). Thus, alternative treatments are needed. One possibility is the use of tissue engineering techniques to repair or replace the degenerated disc tissue, using autologous mesenchymal stem cells (MSCs) (Caplan, 1991) induced to differentiate into a disc-cell phenotype. However, since there is no unique marker for disc tissue, and because hyaline cartilage and immature nucleus pulposus (NP) possess similar macromolecules in their extracellular matrix (Poiraudeau et al., 1999; Sive et al., 2002), it is currently difficult to recognize MSC cell conversion to a disc cell.

Intervertebral discs are composite structures with a peripheral collagen-rich annulus fibrosus (AF) surrounding the proteoglycan-rich central NP (Antoniou et al., 1996b; Hayes et al., 2001). As with articular cartilage, the discs are thought to resist compressive forces by their high content of the proteoglycan aggrecan in the NP (Hutton et al., 2000). In addition, both tissues also contain proteoglycans that have the ability to interact specifically with collagen molecules (Adams et al., 1977; Hayes et al., 2001; Cs-Szabo et al., 2002; Sztrolovics et al., 2002). Intervertebral discs have been reported to differ from articular cartilage in that they contain a significantly higher proportion of the proteoglycan versican (Sztrolovics et al., 2002). With aging, IVD proteoglycans undergo marked changes in their metabolism and composition (Adams and Muir, 1976; Olczyk, 1994), leading to reduction in aggrecan content due to decreased 


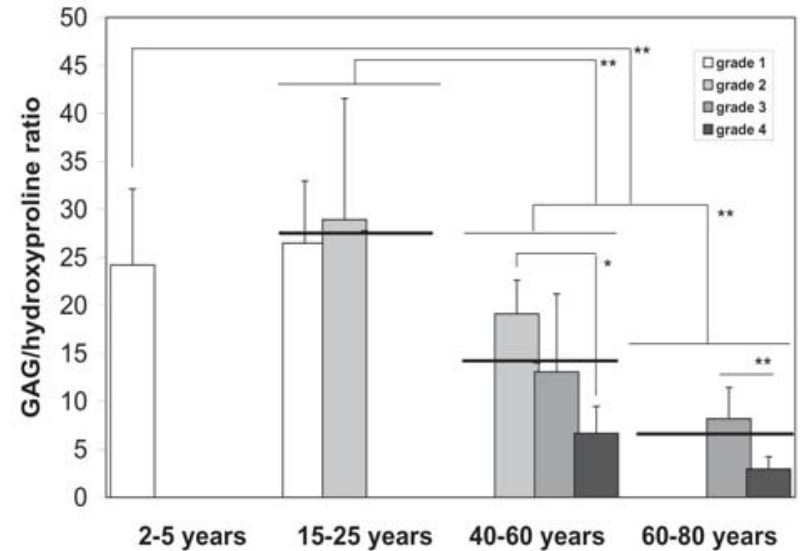

Figure 1. Analysis of GAG to hydroxyproline ratio within the nucleus pulposus related to age. The GAG/ hydroxyproline ratio was determined by dividing GAG ( $\mu \mathrm{g} / \mathrm{mg}$ tissue) by hydroxyproline ( $\mu \mathrm{g} / \mathrm{mg}$ tissue). Mean \pm standard deviation of 8 (2-5 years), 14 (15-25 years), and 17 (40-60 and 60-80 years) samples are shown. *P $<0.0005$ and $* * \mathrm{P}<0.005$. Horizontal bars are the mean GAG/hydroxyproline ratio of the different grades in an age group.

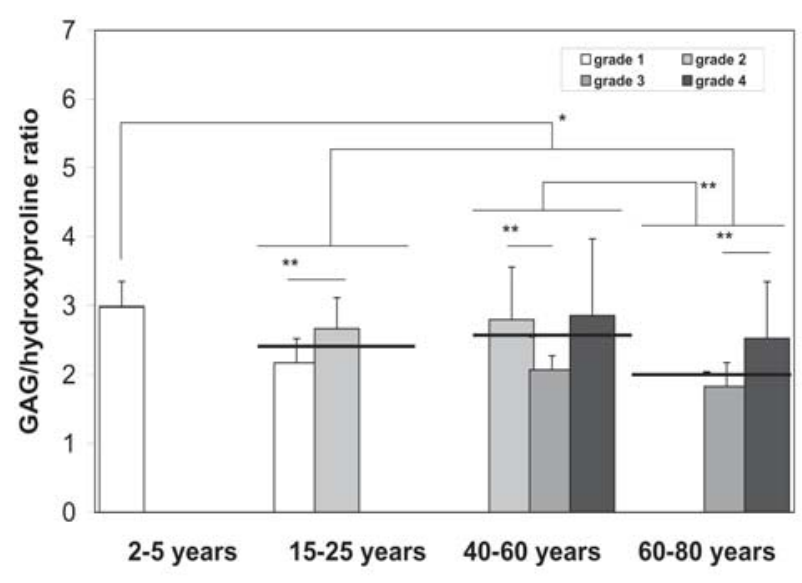

Figure 2. Analysis of GAG to hydroxyproline ratio within hyaline cartilage related to age. The GAG/ hydroxyproline ratio was determined by dividing GAG $(\mu \mathrm{g} / \mathrm{mg}$ tissue) by hydroxyproline $(\mu \mathrm{g} / \mathrm{mg}$ tissue). Mean \pm standard deviation of 8 (2-5 years), 14 (15-25 years), and 17 (40-60 and 60-80 years) samples are shown. $* \mathrm{P}<0.01$ and $* * \mathrm{P}<0.05$. Horizontal bars are the mean GAG/hydroxyproline ratio of the different grades in an age group. proteoglycan synthesis or increased degradation. At least eight distinct collagen types have been identified in the disc: types I, II, III, V, VI, IX, X and XI (Adams et al., 1977; Nerlich et al., 1998). Type II collagen, which is characteristic of hyaline cartilages, is most abundant in the NP (Eyre and Muir, 1976).

In this study, we compare the proteoglycan to collagen ratio in the NP of normal disc to that of the hyaline cartilage of the endplate within the same group of individuals and show that this ratio can be used to distinguish in vivo between a normal NP and hyaline cartilage phenotype. This information is important for ensuring that any NP generated by tissue engineering techniques has appropriate properties for disc function.

\section{Materials and Methods}

\section{Tissue}

Twenty-five whole human lumbar spine specimens were removed from fresh cadavers, ranging in age from 2 to 79 years, and 69 IVDs and adjacent endplates were harvested. Patient details and Thomson grades were summarized previously (Antoniou et al., 1996a; Antoniou et al., 1996b). The discs were separated into the AF and NP zones, as previously described (Antoniou et al., 1996b), and $15 \mathrm{mg}$ of tissue from each region was used for analysis.

\section{Glycosaminoglycans content}

The tissue was solubilized by digestion with proteinase $\mathrm{K}$ in $1 \mathrm{ml}$ buffer (Antoniou et al., 1996b). The resulting digests were used for analysis of the total proteoglycan content as a measure of sulfated glycosaminoglycans (GAG). To measure sulfated GAGs derived predominantly from the proteoglycan aggrecan, $10 \mu \mathrm{l}$ samples of the tissue digests were analyzed using the 1,9 dimethylmethylene blue (DMMB) dye-binding assay (Farndale et al., 1986).

\section{Hydroxyproline content}

The proteinase $\mathrm{K}$ tissue digests were used for analysis of hydroxyproline as a measure of total collagen content. For analysis, digests were first hydrolysed in $6 \mathrm{M} \mathrm{HCl}$ to release free hydroxyproline which was then quantitated using dimethylaminobenzaldehyde (Burleigh et al., 1974). Total collagen content was estimated assuming that hydroxyproline content is equivalent to $10 \%$ of the weight of each collagen $\alpha$ chain (Nimni, 1983).

\section{Statistical analysis}

All values were recorded in a spreadsheet program according to age group, grade of degeneration, and disc region. The GAG/hydroxyproline ratio was determined by dividing GAG ( $\mu \mathrm{g} / \mathrm{mg}$ dry weight) by hydroxyproline $(\mu \mathrm{g} / \mathrm{mg}$ dry weight). The significance of location and degeneration on GAG/hydroxyproline ratio was determined with ANOVA analysis. Significant results were further analyzed using the unpaired Student's t-test. Results were considered significant with $\mathrm{P}<0.05$.

\section{Results}

\section{GAG to hydroxyproline ratio within the NP and hyaline cartilage of the endplate}

In healthy juvenile donors from 2-5 years old, the GAG to hydroxyproline ratio in the NP was on average $25: 1$ (Fig.1). Similarly, in young adult disc with no degeneration (15-25 years), the GAG to hydroxyproline ratio within 


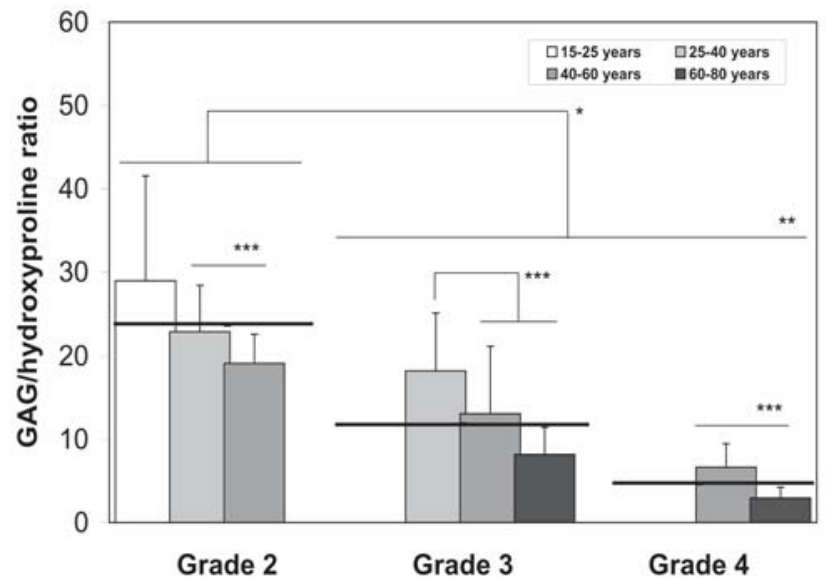

Figure 3. Analysis of GAG to hydroxyproline ratio in the nucleus pulposus related to morphological grade of disc degeneration. The GAG/hydroxyproline ratio was determined by dividing GAG $(\mu \mathrm{g} / \mathrm{mg}$ dry weight) by hydroxyproline $(\mu \mathrm{g} / \mathrm{mg}$ tissue $)$. Mean \pm standard deviation of 21 (grade 2), 24 (grade 3), and 9 (grade 4) samples are shown. $* \mathrm{P}<0.0001, * * \mathrm{P}<0.001$, and $* * * \mathrm{P}<$ 0.05 . Horizontal bars are the mean GAG/hydroxyproline ratio of the different ages in a grade group.

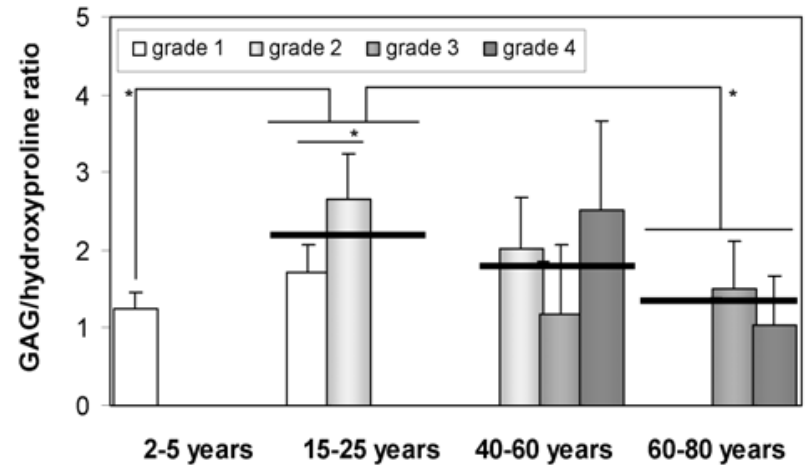

Figure 5. Analysis of GAG to hydroxyproline ratio within the annulus fibrosus related to age. The GAG/ hydroxyproline ratio was determined by dividing GAG ( $\mu \mathrm{g} / \mathrm{mg}$ tissue) by hydroxyproline ( $\mu \mathrm{g} / \mathrm{mg}$ tissue). Mean \pm standard deviation of 8 (2-5 years), 14 (15-25 years), and 17 (40-60 and 60-80 years) samples are shown. $* \mathrm{P}<0.005$. Horizontal bars are the mean GAG/ hydroxyproline ratio of the different grades in an age group.

the NP is approximately $27: 1$. With increasing age there was a marked drop in the GAG to hydroxyproline ratio, which declined to about 5:1 in the elderly (60-80 years).

In comparison, the GAG to hydroxyproline ratio within the hyaline cartilage endplate of the same group of young adults (15-25 years) was about 2:1 (Fig. 2). Depending on age, the GAG to hydroxyproline ratio in the hyaline cartilage varied between $3: 1$ and $2: 1$.

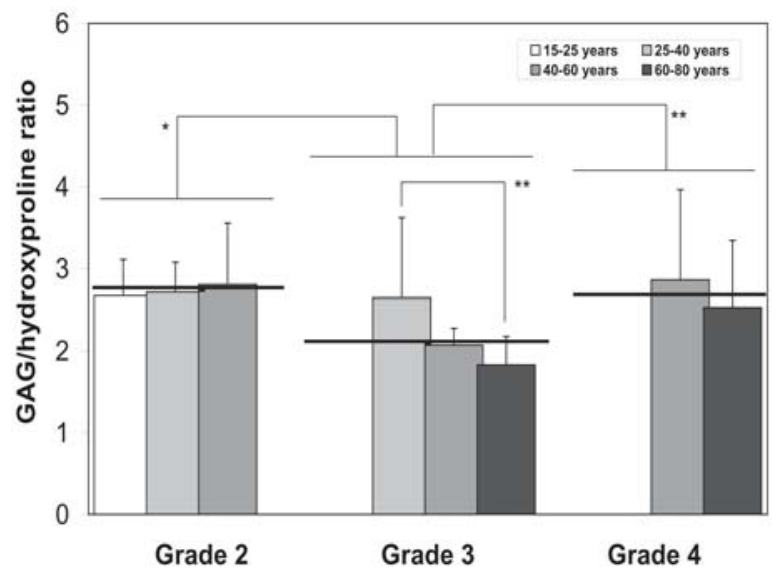

Figure 4. Analysis of GAG to hydroxyproline ratio in the endplate related to morphological grade of disc degeneration. The GAG/hydroxyproline ratio was determined by dividing GAG ( $\mu \mathrm{g} / \mathrm{mg}$ tissue) by hydroxyproline $(\mu \mathrm{g} / \mathrm{mg}$ tissue $)$. Mean \pm standard deviation of 21 (grade 2), 24 (grade 3), and 9 (grade 4) samples are shown. ${ }^{*} \mathrm{P}<0.001$ and $* * \mathrm{P}<0.05$. Horizontal bars are the mean GAG/hydroxyproline ratio of the different ages in a grade group.

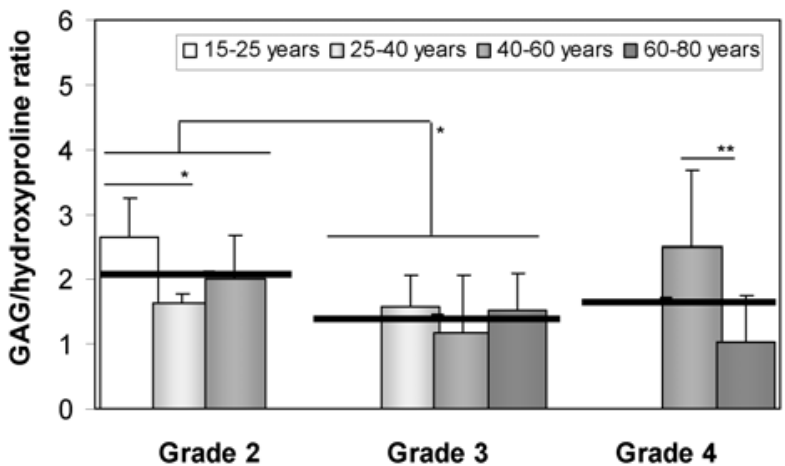

Figure 6. Analysis of GAG to hydroxyproline ratio in the annulus fibrosus related to morphological grade of disc degeneration. The GAG/hydroxyproline ratio was determined by dividing GAG ( $\mu \mathrm{g} / \mathrm{mg}$ tissue) by hydroxyproline $(\mu \mathrm{g} / \mathrm{mg}$ tissue). Mean \pm standard deviation of 21 (grade 2), 24 (grade 3), and 9 (grade 4) samples are shown. $* \mathrm{P}<0.005$ and $* * \mathrm{P}<0.05$. Horizontal bars are the mean GAG/hydroxyproline ratio of the different ages in a grade group.

\section{GAG to hydroxyproline within the NP and cartilage endplate of degenerate discs}

Since aging of the disc is associated with increased degeneration, the GAG to hydroxyproline ratio of the NP (Fig. 3) and endplate (Fig. 4) were also correlated with this parameter. The ratio of the GAG to hydroxyproline in the NP of degenerated discs correlated to gross morphologic Thompson grade was markedly reduced with increasing grade. The GAG to hydroxyproline ratio was highest in patients with grade 2 discs $(\sim 23: 1)$ and was lowest in those with grade 4 $\operatorname{discs}(\sim 5: 1)$. 
The ratios of the GAG to hydroxyproline in the endplate of degenerated discs were correlated to gross morphologic Thompson grade. Independent of grade, the ratio was consistently lower in the endplate (Fig. 4) than in the NP (Fig. 3) ( $\mathrm{P}<0.0001)$. Further, in contrast to the NP, the ratio in the endplate changed only slightly with grade. The lowest ratios were observed in grade 3 discs.

\section{GAG to hydroxyproline ratio within the AF}

The GAG to hydroxyproline ratio was also examined in the AF of the same donors, where it was in all individuals on average 1.6:1 (Fig. 5). Independent of age, the ratio was consistently lower in the AF (Fig. 5) than in the NP (Fig. 1) $(\mathrm{P}<0.0001)$. Further, in contrast to the NP, the ratio in the $\mathrm{AF}$ varied little with age with the highest ratios observed in the young adult disc (15-25 years).

The ratios of the GAG to hydroxyproline in the annulus of degenerated discs were correlated to gross morphologic Thompson grade. Independent of grade, the ratio was consistently lower in the annulus (Fig. 6) than in the NP (Fig. 3) $(\mathrm{P}<0.0001)$.

\section{Discussion}

The NP has often been considered as just another cartilaginous tissue. This is because hyaline cartilage and healthy NP possess similar macromolecules in their extracellular matrix (Adams et al., 1977; Hayes et al., 2001; Sztrolovics et al., 2002), although some differences in the structure of proteoglycans in cartilage and the NP tissues have been observed (Buckwalter et al., 1989).

In this work, GAG and hydroxyproline assays were used to assess the ratio of GAG to hydroxyproline as a means of distinguishing between the disc and cartilage phenotypes. Our findings reveal that in a normal young adult disc (15-25 years old) the GAG to hydroxyproline ratio within the NP is approximately $27: 1$. However, the GAG to hydroxyproline ratio within the hyaline cartilage of the endplate of the same group is about 2:1. This raised the question of whether this difference is unique to the endplate or common to all hyaline cartilages. Recent evidence was presented indicating that human nasal septal cartilage from young (33.7 \pm 3.9 years), middle-aged $(50.2 \pm 5.4$ years $)$ and aged (73.5 \pm 5.8 years) adults had GAG/hydroxyproline ratios of $3.5,3.3$ and 2.5, respectively (Homicz et al., 2003). Similarly, others have found that in subjects aged 14 to 45 years the GAG/ hydroxyproline ratio in talar ankle cartilage, distal femur knee cartilage, and tibial plateau knee cartilage is 1.8, 1.3 and 1.3, respectively (Treppo et al., 2000). Thus, while the precise GAG/hydroxyproline ratio may vary between cartilage sites, it is always below 5 for all hyaline cartilages, and thus always distinct from the values in the NP. These ratios, therefore, act as a useful distinction between the normal NP phenotype and that of hyaline cartilage. In older adults this tissue difference is not so apparent, as the GAG to hydroxyproline ratio progressively decreased with increasing age in the NP due to disc degeneration. However, for tissue engineering purposes one wishes to mimic the properties of the healthy adult disc and not those of the degenerate disc.

The production of a high GAG to hydroxyproline (proteoglycan to collagen) ratio may provide one means of identifying in vivo a NP-like phenotype when stem cells are induced to differentiate into a NP cell as opposed to a chondrocyte or AF cell, for tissue engineering of IVD. Ideally, the GAG content should reflect aggrecan and the hydroxyproline content should reflect type II collagen, as these molecules are pivotal in the ability of the tissue to resist compression. However, aggrecan and type II collagen are unlikely to be sufficient alone. Other proteoglycans such as decorin, biglycan, fibromodulin, lumican and versican, as well as other collagen types and noncollagenous proteins will probably be needed, both to maintain the integrity of the matrix and to ensure the continued ability of the cells to produce an appropriate matrix composition. Although these other molecules are present in relatively low concentrations in the disc, they may be important for tissue engineering purposes if they influence the distinctive functional properties of the NP. Future studies will be needed to address their significance.

A high ratio of GAG:hydroxyproline is a reflection of proteoglycan to collagen content, though the two ratios are not numerically equivalent. GAG may reflect the bulk of proteoglycans (particularly if it is mainly aggrecan), whereas hydroxyproline reflects a low fraction of the total collagen. For example, if one accepts that hydroxyproline content is equivalent to $10 \%$ of the weight of each collagen alpha chain (Nimni, 1983), then for a ratio above 10, there is more proteoglycan than total collagen, whereas for a ratio below 10 , there is more total collagen than proteoglycan. Thus the present results indicate that juvenile and normal adult NP always contain more proteoglycan than collagen, whereas the reverse is true for all hyaline cartilages. In the more degenerate disc, this excess of proteoglycan over collagen is no longer apparent. Other studies have also shown that the GAG:collagen ratio significantly decreases in the NP of prolapsed discs (Olczyk, 1994).

The high proteoglycan to collagen ratio helps explain why the young NP has a gelatinous consistency rather than the firm texture of a hyaline cartilage, as low collagen concentrations are more compatible with a fluid rather than a solid consistency. While both cartilages and NP are designed to resist compression they are not interchangeable in function. The NP of the IVD is designed to operate within the confines of the AF and the vertebral endplates which maintain its boundaries, whereas articular cartilage is designed to operate in isolation and must maintain its own shape. The increase in collagen content is essential for maintenance of cartilage shape, but is not required or desired in the NP. Certainly it is not desirable to generate a NP with a low proteoglycan to collagen ratio that is either firm as hyaline cartilage or fibrous as the AF, as the gelatinous texture of the young NP is ideal for transmitting load in three dimensions when confined by surrounding tissue. Attaining this gelatinous texture and hence producing a high GAG to hydroxyproline ratio in vivo should be the goal when trying to repair or generate a NPlike tissue. 
The production of an extracellular matrix with a high GAG to hydroxyproline ratio can be used to distinguish an appropriate NP generated by tissue engineering techniques from other cartilaginous phenotypes that may be inappropriate for function in the disc, even though all these tissues are capable of resisting compression in their normal physiological role. It is tempting to speculate that it may not matter if one generates authentic disc cells as long as they generate an appropriate extracellular matrix. However, it is important to note that the intervertebral disc is the largest avascular tissue in the body and supports a very low number of cells per unit volume (Urban and Roberts, 2003), that must be adapted to the low oxygen tension (Bartels et al., 1998; Ishihara and Urban, 1999), low glucose concentrations (Maroudas et al., 1975), low $\mathrm{pH}$ (Ohshima and Urban, 1992), and other conditions that are not conducive to high levels of protein synthesis required during tissue repair or regeneration. It is possible that an authentic disc cell might be the only cell capable of generating such a matrix under these adverse conditions.

The source of cells for disc tissue engineering is not immediately apparent, as there is no benign site for harvesting authentic autologous disc cells. It is also preferable to avoid the use of allogeneic donor disc cells. While the avascular nature of the disc may make the NP an immunologically privileged site and therefore make the use of allogeneic cells a tempting proposition, the risk of transferring infectious agents remains real. Thus there is a need to generate disc cells from another autologous source. One possibility is the use of MSCs. If stem cells are to be differentiated into a disc cell phenotype, it will be essential to verify that the ultimate matrix that they produce in vivo has an appropriate proteoglycan to collagen ratio. While this ratio may not help in determining whether appropriate differentiation has occurred, it will be a marker for the correct consistency to the tissue that they produce.

\section{Acknowledgements}

This study was funded by the Canadian Institutes of Health Research, the AO Foundation (Switzerland) (to J.A. and F.M.) the Canadian Orthopaedic Foundation (to F.M.) and the Shriners of North America (to P.R.). We would like to thank Caroline N. Demers for assistance with statistical analysis.

\section{References}

Adams P, Muir H (1976) Qualitative changes with age of proteoglycans of human lumbar discs. Ann Rheum Dis 35: 289-296.

Adams P, Eyre DR, Muir H (1977) Biochemical aspects of development and ageing of human lumbar intervertebral discs. Rheumatol Rehabil 16: 22-29.

Antoniou J, Goudsouzian NM, Heathfield TF, Winterbottom N, Steffen T, Poole AR, Aebi M, Alini M (1996a) The human lumbar endplate. Evidence of changes in biosynthesis and denaturation of the extracellular matrix with growth, maturation, aging, and degeneration. Spine 21: 1153-1161.
Antoniou J, Steffen T, Nelson F, Winterbottom N, Hollander AP, Poole RA, Aebi M, Alini M (1996b) The human lumbar intervertebral disc: evidence for changes in the biosynthesis and denaturation of the extracellular matrix with growth, maturation, ageing, and degeneration. J Clin Invest 98: 996-1003.

Bartels EM, Fairbank JC, Winlove CP, Urban JP (1998) Oxygen and lactate concentrations measured in vivo in the intervertebral discs of patients with scoliosis and back pain. Spine 23: 1-8.

Buckwalter JA, Smith KC, Kazarien LE, Rosenberg LC, Ungar R (1989) Articular cartilage and intervertebral disc proteoglycans differ in structure: an electron microscopic study. J Orthop Res 7: 146-151.

Burleigh MC, Barrett AJ, Lazarus GS. 1974. Cathepsin B1. A lysosomal enzyme that degrades native collagen. Biochem J 137: 387-398.

Caplan AI (1991) Mesenchymal stem cells. J Orthop Res 9: 641-650.

Cinotti G, De Santis P, Nofroni I, Postacchini F (2002) Stenosis of lumbar intervertebral foramen: anatomic study on predisposing factors. Spine 27: 223-229.

Cs-Szabo G, Ragasa-San Juan D, Turumella V, Masuda K, Thonar EJ, An HS (2002) Changes in mRNA and protein levels of proteoglycans of the anulus fibrosus and nucleus pulposus during intervertebral disc degeneration. Spine 27: 2212-2219.

Eyre DR, Muir H (1976) Types I and II collagens in intervertebral disc. Interchanging radial distributions in annulus fibrosus. Biochem J 157: 267-270.

Farndale RW, Buttle DJ, Barrett AJ (1986) Improved quantitation and discrimination of sulphated glycosaminoglycans by use of dimethylmethylene blue. Biochim Biophys Acta 883: 173-177.

Hayes AJ, Benjamin M, Ralphs JR (2001) Extracellular matrix in development of the intervertebral disc. Matrix Biol 20: 107-121.

Homicz MR, McGowan KB, Lottman LM, Beh G, Sah RL, Watson D (2003) A compositional analysis of human nasal septal cartilage. Arch Facial Plast Surg 5: 53-58.

Hutton WC, Ganey TM, Elmer WA, Kozlowska E, Ugbo JL, Doh ES, Whitesides TE, Jr (2000) Does longterm compressive loading on the intervertebral disc cause degeneration? Spine 25: 2993-3004.

Ishihara H, Urban JP (1999) Effects of low oxygen concentrations and metabolic inhibitors on proteoglycan and protein synthesis rates in the intervertebral disc. $\mathrm{J}$ Orthop Res 17: 829-835.

Lee CK (1988) Accelerated degeneration of the segment adjacent to a lumbar fusion. Spine 13: 375-377.

Macfarlane GJ, Thomas E, Croft PR, Papageorgiou AC, Jayson MI, Silman AJ (1999) Predictors of early improvement in low back pain amongst consulters to general practice: the influence of pre-morbid and episoderelated factors. Pain 80: 113-119.

Maroudas A, Stockwell RA, Nachemson A, Urban J (1975) Factors involved in the nutrition of the human lumbar intervertebral disc: cellularity and diffusion of glucose in vitro. J Anat 120: 113-130.

Nakano N, Tomita T (1980) Results of surgical 
treatment of low back pain: a comparative study of the anterior and posterior approach. Int Orthop 4: 101-106.

Nerlich AG, Boos N, Wiest I, Aebi M (1998) Immunolocalization of major interstitial collagen types in human lumbar intervertebral discs of various ages. Virchows Arch 432: 67-76.

Nimni ME (1983) Collagen: structure, function, and metabolism in normal and fibrotic tissues. Semin Arthritis Rheum 13: 1-86.

Ohshima H, Urban JP (1992) The effect of lactate and $\mathrm{pH}$ on proteoglycan and protein synthesis rates in the intervertebral disc. Spine 17: 1079-1082.

Olczyk K (1994) Age-related changes in proteoglycans of human intervertebral discs. Z Rheumatol 53: 19-25.

Poiraudeau S, Monteiro I, Anract P, Blanchard O, Revel M, Corvol MT (1999) Phenotypic characteristics of rabbit intervertebral disc cells. Comparison with cartilage cells from the same animals. Spine 24: 837-844.

Salminen JJ, Erkintalo MO, Pentti J, Oksanen A, Kormano MJ (1999) Recurrent low back pain and early disc degeneration in the young. Spine 24: 1316-1321.

Seitsalo S, Schlenzka D, Poussa M, Osterman K (1997) Disc degeneration in young patients with isthmic spondylolisthesis treated operatively or conservatively: a long-term follow-up. Eur Spine J 6: 393-397.

Sive JI, Baird P, Jeziorsk M, Watkins A, Hoyland JA, Freemont AJ (2002) Expression of chondrocyte markers by cells of normal and degenerate intervertebral discs. Mol Pathol 55: 91-97.

Snider RK, Krumwiede NK, Snider LJ, Jurist JM, Lew RA, Katz JN (1999) Factors affecting lumbar spinal fusion. J Spinal Disord 12: 107-114.

Sztrolovics R, Grover J, Cs-Szabo G, Shi SL, Zhang Y, Mort JS, Roughley PJ (2002) The characterization of versican and its message in human articular cartilage and intervertebral disc. J Orthop Res 20: 257-266.

Treppo S, Koepp H, Quan EC, Cole AA, Kuettner KE, Grodzinsky AJ (2000) Comparison of biomechanical and biochemical properties of cartilage from human knee and ankle pairs. J Orthop Res 18:739-748.

Urban JP, Roberts S (2003) Degeneration of the intervertebral disc. Arthritis Res Ther 5: 120-130.

\section{Discussion with Reviewers}

M.Alini: Although ratio is an important parameter, the authors should point out to the reader that the appropriate molecules (PG and collagen types) as well as biomechanical properties should also be considered. Please comment.

Authors: The production of a high GAG to hydroxyproline (proteoglycan to collagen) ratio may provide one means of identifying in vivo a NP-like phenotype when stem cells are induced to differentiate into a NP cell as opposed to a chondrocyte or AF cell, for tissue engineering of IVD. Ideally, the GAG content should reflect aggrecan and the hydroxyproline content should reflect type II collagen, as these molecules are pivotal in the ability of the tissue to resist compression. However, aggrecan and type II collagen are unlikely to be sufficient alone. Other proteoglycans such as decorin, biglycan, fibromodulin, lumican and versican as well as other collagen types and noncollagenous proteins will probably be needed, both to maintain the integrity of the matrix and to ensure the continued ability of the cells to produce an appropriate matrix composition. Although these other molecules are present in relatively low concentrations in the disc, they may be important for tissue engineering purposes if they influence the distinctive functional properties of the NP. Future studies will be needed to address their significance.

A. Hollander: What other markers might be investigated that would identify nucleus pulposus? Have you considered a gene array approach, comparing NP cells with articular chondrocytes? Quantitative PCR analysis could be used to identify these cells if appropriate markers can be found. Authors: We agree that other markers might be useful to identify the nucleus pulposus cells. As suggested by the reviewer, one approach could be to use gene array analysis, but this is not a trivial quest as gene expression by the NP cells varies with age. NP tissue from a juvenile may yield results that are affected by the presence of notochordal cells, whereas tissue from the mature adult may be influenced by degeneration. It is possible that NP cells from the adolescent may be most appropriate to determine unique markers, other than the GAG:hydroxyproline ratio, for tissue engineering of the NP. Such markers are essential if one is to identify the NP cell itself, rather than the matrix that it produces.

T. Kirsch: Was the tissue used to compare the GAG to hydroxyproline ratio in NP from young and old people healthy or degenerated? If the tissue from the older donors was degenerated what would be the ratio if just healthy tissue from older donors was used?

Authors: Healthy tissue (grade 1 or 2) was observed mainly in the $2-5$ years old ( $100 \%$, grade 1$)$ and in most of the $15-25$ years old (50\% grade 1 and $50 \%$ grade 2$) \mathrm{NP}$ tissues. Only $41 \%$ of NP tissues were of grade 2 in the 40 60 years old, while all discs of the $60-80$ year olds were either grade 3 or 4 (See Fig. 1). The GAG to hydroxyproline ratio of just healthy tissue from the 40-60 years old donors would be about 19:1.

T. Kirsch: What is the age range of the donors whose tissue was compared in Figure 3?

Authors: Two thirds of grade 2 NP tissues were from donors less than 40 years old while the rest were from 40 60 years old. In the grade 3 NP tissue, only $25 \%$ of NP tissues are from those less than 40 years old and half from 60-80 years with the rest between 40 and 60 years old. Forty four percent of grade 4 NP tissues were from 40-60 years old and $56 \%$ from $60-80$ years old.

T. Kirsch: Can the authors comment on the composition of the extracellular matrix of NP and changes during aging (alterations in collagen types or alterations of the expression and degradation of large and small proteoglycans with aging)?

Authors: The extracellular matrix of the NP is composed mainly of proteoglycans, collagens and other noncollogenous proteins. These molecules can undergo 
marked changes in their abundance and structure with age. The most marked changes occur in aggrecan-related proteoglycans whose size decreases and whose keratan sulphate content increases reflecting variation in both synthesis and degradation. The aggrecan degradation products accumulate in the disc extracellular matrix and result in a high tissue content of large non-aggregating proteoglycans. The disc contains numerous collagen types whose relative abundance varies with age. In addition, agerelated glycation of the collagen molecules can result in increased concentrations of pyridinoline and pentosidine cross-links. At present it is unclear whether the age-related changes are beneficial or detrimental to disc function. Certainly, any tissue engineered disc would like to mimic the matrix structure that is optimal for disc function.

I. Stokes: The challenge posed by the authors is to identify a cell phenotype that can synthesize a matrix having an appropriate composition, and then, after implantation, maintain that composition (homeostasis). This practical problem therefore includes two quite differing challenges.
The present work identifies the correct (or desirable) composition requirement, but it is not clear how that composition would be maintained in vivo by the same cell types. Notably, the intervertebral disc, being avascular, supports a very low number of cells per unit volume, and these cells must be adapted to the low oxygen and glucose concentrations, low $\mathrm{pH}$, and other conditions that are not conducive to high levels of protein synthesis required during the tissue engineering phase. They state in the Discussion that 'it may not matter if one generates authentic disc cells. Given the multiple requirements to synthesize and then maintain tissue phenotype in the specific avascular environment of the disc, is it likely that anything but 'authentic' cells would be able to perform satisfactorily? Authors: We agree with the reviewer. It is tempting to speculate that it may not matter if one generates authentic disc cells as long as they generate an appropriate extracellular matrix. However, it is possible that an authentic disc cell might be the only cell capable of generating such a matrix under these adverse conditions. 